QCD Evolution Workshop: From Collinear to Non-Collinear Case International Journal of Modern Physics: Conference Series Vol. 4 (2011) 157-167

(C) World Scientific Publishing Company

DOI: $10.1142 / \mathrm{S} 2010194511001668$

\title{
EVOLUTION EQUATIONS OF TWIST-THREE PARTON DISTRIBUTIONS
}

\author{
BJÖRN PIRNAY \\ Institut für Theoretische Physik, Universität Regensburg, \\ Regensburg, D-93040, Germany \\ bjoern.pirnay@physik.uni-regensburg.de
}

\begin{abstract}
We report on a recent work concerning the scale dependence of twist-three correlation functions relevant for the single transverse spin asymmetry in the framework of collinear factorization. Evolution equations are presented for both the flavor-nonsinglet and flavor-singlet distributions. Our results do not agree with previous calculations of the evolution in the limit of vanishing gluon momentum. Possible sources for this discrepancy are identified.
\end{abstract}

Keywords: Evolution equations; higher twist; spin asymmetries.

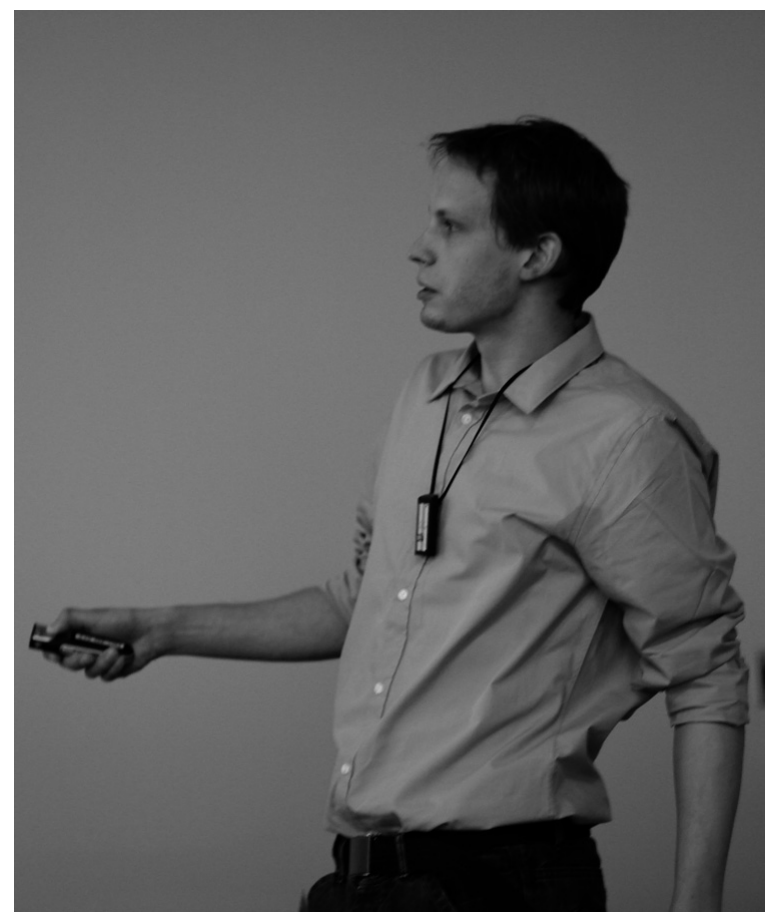




\section{Introduction}

Large transverse single spin asymmetries (SSAs) have been observed in different hadronic reactions and these observations generated a lot of interest. Such experiments are conceptually rather simple, but their theoretical description proved to be challenging as the leading-twist contributions to such asymmetries vanish, see e.g. Ref. [1] for a review. Over the past few years there was a splash of theoretical activity in this field, which mainly followed two lines: the $k_{\perp}$ factorization in terms of the transverse-momentum dependent (TMD) distributions, or, alternatively, collinear factorization including twist-three contributions in terms of multiparton correlation functions ${ }^{2,3,4}$. These two techniques have their own domain of validity and were shown to be consistent with each other in the kinematic regime where they both $\operatorname{apply}^{5,6,7,8}$.

However, practically all existing calculations have so far been at the leading order (LO) which corresponds, roughly speaking, to the (generalized) parton model. In order to test QCD dynamics and eventually also reduce the dependence of theory predictions on both the factorization scale and the renormalization scale of the strong coupling, it is necessary to calculate the scale dependence (evolution) of the relevant nonperturbative functions. This calculation was addressed in Refs. $[9,10]$ where the evolution equation was derived for the gluonic pole contributions to twistthree correlation functions that are relevant to SSA. Unfortunately, it turns out that the equations derived in $[9,10]$ are in contradiction to earlier results obtained in the framework of the operator product expansion (OPE). A resolution of this discrepancy presents the main motivation for this study.

Another question that we want to address is to clarify the relation of these results to earlier calculations of the scale dependence of twist-three correlation functions that contribute to inclusive reactions, pioneered by the study of the structure function $g_{2}\left(x, Q^{2}\right)$ in Ref. [11]. We would like to emphasize that the scale dependence of an arbitrary twist-three operator in QCD, hence arbitrary twist-three light-cone correlation function can be determined in terms of the two-particle evolution kernels introduced by Bukhvostov, Frolov, Lipatov and Kuraev (BFLK) ${ }^{12}$. The evolution equations for the particular parton distributions relevant for SSA can be obtained from the BFLK kernels by simple algebra. Using an updated version of this technique ${ }^{13,14}$ we write down the complete evolution equations for all relevant three-particle correlation functions.

\section{Relevant Twist-3 Operators, Light-cone Correlation Functions and Their Properties}

Following Ref. [9] we will consider the correlation functions, corresponding to the nucleon matrix elements of the quark-antiquark-gluon and three-gluon light-ray 
operators

$$
\begin{aligned}
T_{\mu}\left(z_{1}, z_{2}, z_{3}\right) & =g \bar{q}\left(z_{1} n\right) \gamma_{+} F_{\mu+}\left(z_{2} n\right) q\left(z_{3} n\right), \\
\Delta T_{\mu}\left(z_{1}, z_{2}, z_{3}\right) & =g \bar{q}\left(z_{1} n\right) \gamma_{+} \gamma_{5} i F_{\mu+}\left(z_{2} n\right) q\left(z_{3} n\right), \\
G_{\mu \rho \lambda}^{ \pm}\left(z_{1}, z_{2}, z_{3}\right) & =g C_{ \pm}^{a b c} F_{+\rho}^{a}\left(z_{1} n\right) F_{+\mu}^{b}\left(z_{2} n\right) F_{+\lambda}^{c}\left(z_{3} n\right) .
\end{aligned}
$$

Here $n_{\mu}$ is a light-like vector, $n^{2}=0$, the "plus" projection is defined as $a_{+}=a^{\mu} n_{\mu}$. Note that there are two gluon operators with a different color structure; the factors $C_{ \pm}^{a b c}$ are written in terms of the $S U(3)$ structure constants

$$
C_{+}^{a b c}=i \mathrm{f}^{a b c}, \quad C_{-}^{a b c}=\mathrm{d}^{a b c} .
$$

In all cases the path-ordered Wilson lines are implied that ensure gauge invariance.

For our purposes it is convenient to define the relevant twist-3 correlation functions in a symmetric notation with quark, antiquark and gluon momentum fractions treated equally

$$
\begin{aligned}
\left\langle P, s_{T}\left|\tilde{s}^{\mu} T_{\mu}\left(z_{1}, z_{2}, z_{3}\right)\right| P, s_{T}\right\rangle & =2 P_{+}^{2} \int \mathcal{D} x e^{-i P_{+} \sum_{k} x_{k} z_{k}} T_{\bar{q} F q}\left(x_{1}, x_{2}, x_{3}\right), \\
\left\langle P, s_{T}\left|s^{\mu} \Delta T_{\mu}\left(z_{1}, z_{2}, z_{3}\right)\right| P, s_{T}\right\rangle & =2 P_{+}^{2} \int \mathcal{D} x e^{-i P_{+} \sum_{k} x_{k} z_{k}} \Delta T_{\bar{q} F q}\left(x_{1}, x_{2}, x_{3}\right), \\
g^{\rho \lambda}\left\langle P, s_{T}\left|\tilde{s}^{\mu} G_{\mu \rho \lambda}^{ \pm}\left(z_{1}, z_{2}, z_{3}\right)\right| P, s_{T}\right\rangle & =P_{+}^{3} \int \mathcal{D} x e^{-i P_{+} \sum_{k} x_{k} z_{k}} T_{3 F}^{ \pm}\left(x_{1}, x_{2}, x_{3}\right), \\
\epsilon_{\perp}^{\rho \lambda}\left\langle P, s_{T}\left|\tilde{s}^{\mu} G_{\mu \rho \lambda}^{ \pm}\left(z_{1}, z_{2}, z_{3}\right)\right| P, s_{T}\right\rangle & =P_{+}^{3} \int \mathcal{D} x e^{-i P_{+} \sum_{k} x_{k} z_{k}} \Delta T_{3 F}^{ \pm}\left(x_{1}, x_{2}, x_{3}\right)
\end{aligned}
$$

where the integration measure is defined as

$$
\int \mathcal{D} x=\int_{-1}^{1} d x_{1} d x_{2} d x_{3} \delta\left(x_{1}+x_{2}+x_{3}\right)
$$

and $s_{\mu}$ is the nucleon spin vector normalized by the condition $s^{2}=-1, \tilde{s}^{\mu}=$ $-\epsilon^{\mu \nu \rho \sigma} s_{\nu} n_{\rho} \tilde{n}_{\sigma}$ with $\tilde{n}$ being a second light-like vector, $\tilde{n}^{2}=0, n \tilde{n}=1$ and $\epsilon_{\perp}^{\rho \lambda}=$ $-\epsilon^{\rho \lambda n \tilde{n}}, \epsilon_{0123}=1$. The spin vector $s_{\mu}$ is assumed to be transverse, $n s=\tilde{n} s=0$. We assume here the standard (relativistic) normalization of states; the factor $P_{+}^{2}$ is necessary to ensure the reparametrization invariance to the choice of the light-cone vector $n_{\mu} \rightarrow \alpha n_{\mu}$.

The conversion to the distribution $\mathcal{T}_{q, F}$ introduced in Ref. [9] is achieved by

$$
\mathcal{T}_{q, F}\left(x, x+x_{2}\right) \equiv T_{\bar{q} F q}\left(-x-x_{2}, x_{2}, x\right),
$$

and similarly for the other distributions.

Writing the definition in such a (Fourier-transformed) form makes the support properties of the correlation functions explicit: they are defined on the surface $x_{1}+$ $x_{2}+x_{3}=0$ and effectively are functions of two variables only. These correlation functions vanish for any momentum fraction variable $\left|x_{i}\right|>1$. A nice graphical representation of this support can be made by using a analogue to barycentric coordinates as shown in Fig. 1:

$$
\vec{x}=x_{1} \vec{e}_{1}+x_{2} \vec{e}_{2}+x_{3} \vec{e}_{3}=x_{1} \vec{E}_{1}+x_{2} \vec{E}_{2} .
$$




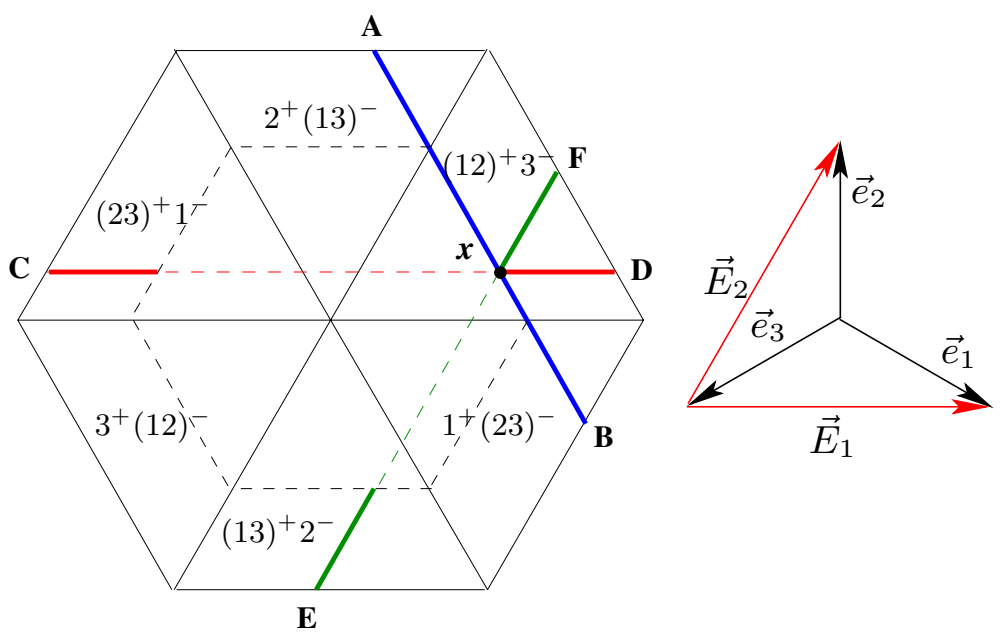

Fig. 1. (Color online) Support properties and schematic evolution of the correlation functions (3) in barycentric coordinates. For the explanation of different regions and lines see text.

The correlation functions "live" on a hexagonal simplex, in which one can classify six different regions, labeled $(12)^{+} 3^{-}, 2^{+}(13)^{-}$, etc., corresponding to different subprocesses at the parton level ${ }^{15}$; For each parton $k=1,2,3$ "plus" stands for emission $\left(x_{k}>0\right)$ and "minus" for absorption $\left(x_{k}<0\right)$. Alternatively, one may think of "plus" and "minus" labels as indicating whether the parton appears in the direct or the final amplitude in the cut diagram ${ }^{16}$.

It is important that different regions do not have autonomous scale dependence; they "talk" to each other and get mixed under the evolution. The particular mixing pattern can be understood as follows. It is easy to see that to one loop accuracy any contribution to the evolution equation only involves two of the three partons. For example, parton-1 and parton- 2 can exchange a gluon whereas parton- 3 stays a spectator, etc. The full evolution kernel can, therefore, be split in three two-particle kernels that involve parton pairs (12), (23) and (31), respectively. Schematically

$$
\mu \frac{d}{d \mu} T_{\bar{q} F q}=\frac{\alpha_{s}}{2 \pi}\left[H_{12}+H_{23}+H_{31}\right] \otimes T_{\bar{q} F q} .
$$

Each two-particle kernel is, obviously, a function of the contributing parton momentum fractions, e.g. $H_{12} \equiv H_{12}\left(x_{1}, x_{2} ; x_{1}^{\prime}, x_{2}^{\prime}\right)$ and due to energy conservation $x_{1}^{\prime}+x_{2}^{\prime}=x_{1}+x_{2}$. In other words, the rate for the scale variation of a three-particle parton correlation function with given values of momentum fractions $x_{1}, x_{2}$ can only involve this function on the line of constant $x_{1}+x_{2}=-x_{3}$ (for the contribution of $H_{12}$ ), as shown in Fig. 1 (thick blue line $\mathbf{A B}$ ).

Since $x_{3} \neq 0$ (in general), the total momentum fraction carried by the two participating partons is non-zero, $x_{1}+x_{2} \neq 0$. This situation is familiar from studies of the scale dependence of leading-twist generalized parton distributions (GPDs) (see e.g. $[17,18]$ ) and the three regions $2^{+}(13)^{-},(12)^{+} 3^{-}, 1^{+}(23)^{-}$traversed by the 
thick blue line $\mathbf{A B}$ in Fig. 1 are in one-to-one correspondence to the two DGLAP regions $\left(2^{+} 1^{-}\right.$and $\left.1^{+} 2^{-}\right)$and the ERBL (central) region $\left(1^{+} 2^{+}\right)$in the corresponding evolution equations. As well known ${ }^{17,18}$, the scale dependence of GPDs in the DGLAP regions is autonomous, whereas in the ERBL mode there are also terms describing "leakage" from the DGLAP regions. In the present context, this result implies that the evolution equation for a generic three-particle light-cone correlation function for the momentum fractions $x_{1}, x_{2}$ in the $(12)^{+} 3^{-}$region, as in Fig. 1, will receive nontrivial contributions from the $2^{+}(13)^{-}, 1^{+}(23)^{-}, 2^{-}(13)^{+}$and $1^{-}(23)^{+}$ regions as well, which have a different partonic interpretation.

So far we have considered the contribution of $H_{12}$ only. The contributions of $H_{23}$ and $H_{13}$ are, in turn, kinematically constrained to the lines of constant $x_{2}^{\prime}+x_{3}^{\prime}$ and $x_{1}^{\prime}+x_{3}^{\prime}$, respectively, and, for the particular choice of $x_{1}, x_{2}$ in the $(12)^{+} 3^{-}$ region, correspond to the DGLAP modes of the corresponding GPD-like evolution equations. Recall that the DGLAP evolution is ordered in the momentum fraction; hence only parts of the kinematically allowed regions contribute, as shown in Fig. 1 by the thick red $\mathbf{C D}\left(H_{13}\right)$ and thick green $\mathbf{E F}\left(H_{23}\right)$ lines.

Note that evolution of the parton correlation function for given values of $x_{1}, x_{2}$ only involves the regions of momentum fractions outwards from the center. If one draws a small(er) hexagon (see Fig. 1) inside the big one, then evolution of the correlation function outside of the small hexagon does not depend on the latter inside the small hexagon. This "radial" ordering is a generalization of the usual momentum fraction ordering in DGLAP equations. In contrast, there is no ordering/restrictions in the "azimuthal" direction and, in principle, all regions "talk" to each other. This property may result in the increase of the evolution rate in the small $x$ region.

\section{Evolution Equations}

For our purposes it is convenient to introduce $C$-even/odd combinations of quarkgluon operators (cf. [23] for details)

$$
\begin{aligned}
& \mathfrak{S}^{ \pm}(\boldsymbol{z})=\tilde{s}^{\rho}\left[S_{\rho}^{+}(\boldsymbol{z}) \pm P_{13} S_{\rho}^{-}(\boldsymbol{z})\right], \\
& \mathcal{F}^{ \pm}(\boldsymbol{z})=2 g C_{ \pm}^{a b c} \tilde{s}^{\rho}\left(1 \mp P_{23} \pm P_{12}\right) F_{+}^{a, \nu}\left(z_{1}\right) F_{+\rho}^{b}\left(z_{2}\right) F_{+\nu}^{c}\left(z_{3}\right),
\end{aligned}
$$

where

$$
S_{\rho}^{ \pm}(\boldsymbol{z})=g \bar{q}\left(z_{1}\right)\left[F_{\rho+}\left(z_{2}\right) \pm i \gamma_{5} \tilde{F}_{\rho+}\left(z_{2}\right)\right] \gamma_{+} q\left(z_{3}\right)
$$

\subsection{Flavor-nonsinglet}

The flavor-nonsinglet light-ray operators $\mathfrak{S}_{\mathrm{NS}}^{+}$and $\mathfrak{S}_{\mathrm{NS}}^{-}$satisfy the same evolution equation,

$$
\left(\mu \frac{\partial}{\partial \mu}+\beta(g) \frac{\partial}{\partial g}+\frac{\alpha_{s}}{2 \pi} \mathbb{H}\right) \mathfrak{S}_{\mathrm{NS}}^{ \pm}=0
$$


where $\mathbb{H}$ is an integral operator. The explicit expression for $\mathbb{H}$ (at one-loop) can be restored from the result for $S^{ \pm}$in Ref. [19] (see also [20, 21, 22]). Alternatively, the answer for $\mathbb{H}$ can be obtained by simple algebra from the known expressions for the one-loop two-particle BFLK kernels ${ }^{12,14}$. This technique is quite general and applicable to arbitrary twist-three (and twist-four ${ }^{14}$ ) evolution equations.

After a simple algebra one gets

$$
\mathbb{H}=N_{c} \mathbb{H}_{0}-\frac{1}{N_{c}} \mathbb{H}_{1}-3 C_{F},
$$

where, in the notation of Ref. [14],

$$
\mathbb{H}_{0}=\widehat{\mathcal{H}}_{12}+\widehat{\mathcal{H}}_{23}-2 \mathcal{H}_{12}^{+}, \quad \mathbb{H}_{1}=\widehat{\mathcal{H}}_{13}-\mathcal{H}_{13}^{+}-P_{23} \mathcal{H}_{23}^{e,(1)}+2 \mathcal{H}_{12}^{-} .
$$

Here $\mathcal{H}_{i k}$ are two-particle integral operators that act on the light-cone coordinates of the $i$-th and $k$-th partons:

$$
\left[\mathcal{H}_{i k} \phi\right]\left(z_{i}, z_{k}\right)=\int d z_{i}^{\prime} d z_{k}^{\prime} \mathcal{H}\left(z_{i}, z_{k} \mid z_{i}^{\prime}, z_{k}^{\prime}\right) \phi\left(z_{i}^{\prime}, z_{k}^{\prime}\right) .
$$

These kernels are $S L(2)$-invariant and depend on the conformal spins of partons that they are acting on and the explicit expressions are written down in Ref. [14].

Taking the nucleon matrix element of Eq. (10) one obtains the evolution equation for the corresponding parton distribution function. Technically, this requires a translation from coordinate to momentum fraction space $\left\{z_{1}, z_{2}, z_{3}\right\} \rightarrow\left\{x_{1}, x_{2}, x_{3}\right\}$. Thanks to energy conservation, two-particle kernels in momentum fraction space can be written in the following generic form

$$
\left[\mathcal{H}_{i k} \varphi\right]\left(x_{i}, x_{k}\right)=\int_{-\infty}^{\infty} \mathcal{D} x^{\prime} \mathcal{H}\left(x_{i}, x_{k} \mid x_{i}^{\prime}, x_{k}^{\prime}\right) \varphi\left(x_{i}^{\prime}, x_{k}^{\prime}\right),
$$

where $\mathcal{D} x^{\prime}=d x_{i}^{\prime} d x_{k}^{\prime} \delta\left(x_{i}+x_{k}-x_{i}^{\prime}-x_{k}^{\prime}\right)$. It is assumed that restrictions on integration regions over $x_{1}^{\prime}, x_{2}^{\prime}$ come from support properties of the kernels and the parton distributions; the individual expressions are collected in the Appendix of Ref. [23].

The flavor-nonsinglet "quark-type" correlation functions satisfy the evolution equations

$$
\begin{aligned}
\mu \frac{d}{d \mu} T_{\bar{q} F q}(x) & =-\frac{\alpha_{s}}{4 \pi}\left(\mathbb{H}+P_{13} \mathbb{H} P_{13}\right) T_{\bar{q} F q}(x)+\frac{\alpha_{s}}{4 \pi}\left(\mathbb{H}-P_{13} \mathbb{H} P_{13}\right) \Delta T_{\bar{q} F q}(x), \\
\mu \frac{d}{d \mu} \Delta T_{\bar{q} F q}(x) & =-\frac{\alpha_{s}}{4 \pi}\left(\mathbb{H}+P_{13} \mathbb{H} P_{13}\right) \Delta T_{\bar{q} F q}(x)+\frac{\alpha_{s}}{4 \pi}\left(\mathbb{H}-P_{13} \mathbb{H} P_{13}\right) T_{\bar{q} F q}(x),
\end{aligned}
$$

which is our final result. The "Hamiltonian" $\mathbb{H}$ is defined in Eqs. (11), (12).

To compare our result with the calculation in Ref. [9] we have to change notation to

$$
\mathcal{T}_{q, F}\left(x, x^{\prime}\right) \equiv T_{\bar{q} F q}\left(-x^{\prime}, x^{\prime}-x, x\right), \quad \mathcal{T}_{\Delta q, F}\left(x, x^{\prime}\right) \equiv \Delta T_{\bar{q} F q}\left(-x^{\prime}, x^{\prime}-x, x\right),
$$


which are (anti)symmetric functions under permutation $x \leftrightarrow x^{\prime}, \mathcal{T}_{q, F}\left(x, x^{\prime}\right)=$ $\mathcal{T}_{q, F}\left(x^{\prime}, x\right)$ and $\mathcal{T}_{\Delta, F}\left(x, x^{\prime}\right)=-\mathcal{T}_{\Delta q, F}\left(x^{\prime}, x\right)$. Using explicit expressions for the twoparticle kernels and taking the soft gluon-pole limit $x^{\prime}=x\left(x_{2}=0\right)$ we obtain (it is assumed that $x>0$ )

$$
\begin{aligned}
\mu \frac{d}{d \mu} \mathcal{T}_{q, F}(x, x)= & \frac{\alpha_{s}}{\pi}\left\{-N_{c} \mathcal{T}_{q, F}(x, x)+\int_{x}^{1} \frac{d \xi}{\xi}\left[P_{q q}(z) \mathcal{T}_{q, F}(\xi, \xi)\right.\right. \\
& \left.+\frac{N_{c}}{2}\left(\frac{(1+z) \mathcal{T}_{q, F}(x, \xi)-\left(1+z^{2}\right) \mathcal{T}_{q, F}(\xi, \xi)}{1-z}-\mathcal{T}_{\Delta q, F}(x, \xi)\right)\right] \\
& \left.+\frac{1}{2 N_{c}} \int_{x}^{1} \frac{d \xi}{\xi}\left[(1-2 z) \mathcal{T}_{q, F}(x, x-\xi)-\mathcal{T}_{\Delta q, F}(x, x-\xi)\right]\right\},
\end{aligned}
$$

where $z=x / \xi$,

$$
\begin{aligned}
P_{q q}(z) & =C_{F}\left[\frac{1+z^{2}}{(1-z)_{+}}+\frac{3}{2} \delta(1-z)\right] \\
\int_{x}^{1} d z \frac{f(z)}{(1-z)_{+}} & =\int_{x}^{1} d z \frac{f(z)-f(1)}{1-z}+f(1) \log (1-x) .
\end{aligned}
$$

The partonic interpretation of different contributions in Eq. (17) is illustrated in Fig. 1. Note that the condition of zero gluon momentum $x_{2}=0$ corresponds to the choice of $\vec{x}$ on the (positive) horizontal axis. The term $\sim \mathcal{T}_{q, F}(\xi, \xi)$ on the r.h.s. of Eq. (17) corresponds to the integration over the horizontal line segment $\mathrm{xD}$ shown in red in Fig. 1. The terms involving $\mathcal{T}_{q, F}(x, \xi)$ are due to the integration along the blue, $\mathbf{A B}$, and green, $\mathbf{E F}$, lines in the regions $1^{+}(23)^{-}$and $(12)^{+} 3^{-}$, and the ones containing $\mathcal{T}_{q, F}(x, x-\xi)$ stem from the contributions along the same lines in the regions $2^{+}(13)^{-}$and $(13)^{+} 2^{-}$, respectively. By construction, the function $\mathcal{T}_{q, F}\left(x, x^{\prime}\right)$ is symmetric and $\mathcal{T}_{\Delta q, F}\left(x, x^{\prime}\right)$ antisymmetric under the interchange of arguments, $x \leftrightarrow x^{\prime}$. In Fig. 1 this corresponds to a reflection symmetry around the horizontal axis.

The evolution equation (17) differs from the result by Kang and Qiu (see Eq. (99) in [9]) by the first term in the first line and the term in the third line. The latter term, proportional to $1 / N_{c}$, originates from the kernels $P_{23} \mathcal{H}_{23}^{e,(1)}$ and $\mathcal{H}_{12}^{-}$in Eq. (12). On the diagrammatic level this contribution corresponds to the "exchange" diagrams of the type shown in Fig. 2 which correspond to mixing of the regions that have different partonic interpretation: $2^{+}(13)^{-} \leftrightarrow 1^{+}(23)^{-}$, or $(13)^{+} 2^{-} \leftrightarrow(12)^{+} 3^{-}$. Similar contributions have been discussed in a somewhat different context in Ref. [24].

The other difference is the extra term $-N_{c} \mathcal{T}_{q, F}(x, x)$ in the second line in Eq. (17) for which we do not see any obvious explanation. The origin of this term in our calculation can be traced to the kernels $\widehat{\mathcal{H}}_{12}$ and $\widehat{\mathcal{H}}_{23}$, see Ref. [23]. 


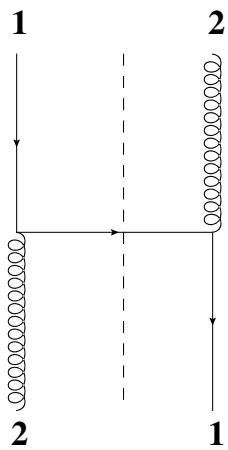

Fig. 2. The exchange diagram.

The evolution does simplify, however, in the large $x$ limit in which case the integration regions shrink to a point. One obtains

$$
\mu \frac{d}{d \mu} \mathcal{T}_{q, F}(x, x)=\frac{\alpha_{s}}{\pi} \int_{x}^{1} \frac{d \xi}{\xi} P_{q, F}^{N S, z \rightarrow 1}(z) \mathcal{T}_{q, F}(\xi, \xi),
$$

where, retaining singular terms at $z \rightarrow 1$ only

$$
P_{q, F}^{N S, z \rightarrow 1}(z)=2 C_{F}\left[\frac{1}{(1-z)_{+}}+\frac{3}{4} \delta(1-z)\right]-N_{c} \delta(1-z) .
$$

This result can be compared with the usual structure function $F_{1}\left(x, Q^{2}\right)$ and the twist-three contribution to the structure function $g_{2}\left(x, Q^{2}\right)^{21,25}$. Their renormalization group kernels are, in this limit, dominated by

$$
\begin{aligned}
& P_{q q}^{N S, z \rightarrow 1}(z)=2 C_{F}\left[\frac{1}{(1-z)_{+}}+\frac{3}{4} \delta(1-z)\right] \\
& P_{g_{2}}^{N S, z \rightarrow 1}(z)=2 C_{F}\left[\frac{1}{(1-z)_{+}}+\frac{3}{4} \delta(1-z)\right]-\frac{N_{c}}{2} \delta(1-z)
\end{aligned}
$$

respectively. The last term proportional to $\delta(1-z)$ is written in the large- $N_{c}$ limit. The contributions $\sim 1 /(1-z)_{+}$are the same in all three cases, which indicates that all three functions $\mathcal{T}_{q, F}\left(x, x ; Q^{2}\right), F_{1}\left(x, Q^{2}\right)$ and $g_{2}^{t w-3}\left(x, Q^{2}\right)$ may have the same functional dependence on the Bjorken variable $x$ in the $x \rightarrow 1$ limit. Different terms $\sim \delta(1-z)$ suggest that, on the other hand, twist-three functions are suppressed at large scales $Q^{2}$ compared to the twist-two distribution:

$$
\frac{\mathcal{T}_{q, F}\left(x, x ; Q^{2}\right)}{F_{1}\left(x, Q^{2}\right)} \sim\left(\frac{\alpha_{s}(Q)}{\alpha_{s}\left(\mu_{0}\right)}\right)^{2 N_{c} / b_{0}} \quad, \quad \frac{g_{2}^{t w-3}\left(x, Q^{2}\right)}{F_{1}\left(x, Q^{2}\right)} \sim\left(\frac{\alpha_{s}(Q)}{\alpha_{s}\left(\mu_{0}\right)}\right)^{N_{c} / b_{0}} .
$$

The suppression of $g_{2}^{t w-3}\left(x, Q^{2}\right)$ compared to $F_{1}\left(x, Q^{2}\right)$ exactly corresponds to the gap between the lowest anomalous dimension in the spectrum of twist-three operators and the usual twist-two anomalous dimension, cf. Fig. 3. For the function $\mathcal{T}_{q, F}\left(x, x ; Q^{2}\right)$ we predict a stronger suppression which translates to a scaling violation in SSA. 


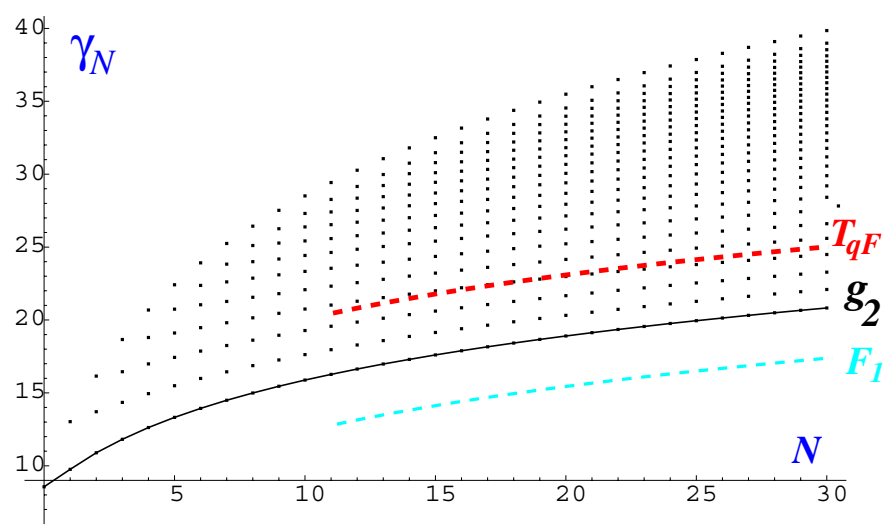

Fig. 3. Anomalous dimensions $\gamma_{N}$ of $\mathcal{T}_{q, F}$ (red), $g_{2}^{t w-3}$ (black) and $F_{1}$ (blue).

\subsection{Flavor-singlet}

In the flavor-singlet sector one has to take into account mixing between the quarkantiquark-gluon and three-gluon operators with the same $C$-parity. Namely, $\mathfrak{S}^{+}$ gets mixed with $\mathcal{F}^{+}$and $\mathfrak{S}^{-}$with $\mathcal{F}^{-}$. For each case, the evolution equation takes the matrix form

$$
\left(\mu \frac{\partial}{\partial \mu}+\beta(g) \frac{\partial}{\partial g}+\frac{\alpha_{s}}{2 \pi} \mathbb{H}^{ \pm}\right)\left(\begin{array}{c}
\mathfrak{S}^{ \pm} \\
\mathcal{F}^{ \pm}
\end{array}\right)=0, \quad \mathbb{H}^{ \pm}=\left(\begin{array}{ll}
\mathbb{H}_{Q Q}^{ \pm} & \mathbb{H}_{Q F}^{ \pm} \\
\mathbb{H}_{F Q}^{ \pm} & \mathbb{H}_{F F}^{ \pm}
\end{array}\right)
$$

In what follows we assume that the quark-antiquark-gluon flavor-singlet operator is defined including the sum over $n_{f}$ light flavors, $\bar{q} F q=\bar{u} F u+\bar{d} F d+\ldots$ The corresponding expressions for the "Hamiltonians" in coordinate space as well as in momentum space are given in Ref. [23].

In the limit of zero gluon momentum our result for the evolution of $T_{q, F}(x, x) \equiv$ $T_{\bar{q} F q}(-x, 0, x)$ differs from the expression in Eq. (107) of Ref. [9] by the same two terms as in the nonsinglet case; the terms in $P_{q g}$ (in our calculation due to $\mathbb{H}_{Q F}$ ) coincide.

To compare our results for three-gluon distributions we introduce the functions

$$
T_{F}^{ \pm}\left(x, x^{\prime}\right)=\frac{1}{x} T_{3 F}^{ \pm}\left(-x^{\prime}, x-x^{\prime}, x\right), \quad \Delta T_{F}^{ \pm}\left(x, x^{\prime}\right)=\frac{1}{x} \Delta T_{3 F}^{ \pm}\left(-x^{\prime}, x-x^{\prime}, x\right),
$$

which coincide with $T_{G, F}^{(f)}\left(x, x^{\prime}\right), T_{G, F}^{(d)}\left(x, x^{\prime}\right), T_{\Delta G, F}^{(f)}\left(x, x^{\prime}\right), T_{\Delta G, F}^{(d)}\left(x, x^{\prime}\right)$ defined in Ref. [9], respectively. 
After some algebra one obtains the following equations for $T_{F}^{ \pm}(x, x)$ :

$$
\begin{aligned}
\mu \frac{d}{d \mu} T_{F}^{ \pm}(x, x)= & \frac{\alpha_{s} N_{c}}{\pi}\left(-T_{F}^{ \pm}(x, x)+\int_{x}^{1} \frac{d \xi}{\xi}\left\{2 \bar{P}_{g g}(z) T_{F}^{ \pm}(\xi, \xi)\right.\right. \\
& +\frac{z}{1-z}\left[T_{F}^{ \pm}(\xi, x)-T_{F}^{ \pm}(\xi, \xi)\right]-(1-z)\left(z+\frac{1}{z}\right) T_{F}^{ \pm}(\xi, \xi) \\
& +\frac{1+z}{2}\left[T_{F}^{ \pm}(x, \xi)-\Delta T_{F}^{ \pm}(x, \xi)\right] \\
& \mp \frac{1}{2}(1-z)\left[T_{F}^{ \pm}(x, x-\xi)-\Delta T_{F}^{ \pm}(x, x-\xi)\right] \\
& \left.\left.+\frac{1}{2} A^{ \pm} \bar{P}_{g q}(z)\left[T_{q, F}(\xi, \xi) \pm T_{q, F}(-\xi,-\xi)\right]\right\}\right)
\end{aligned}
$$

where $A^{+}=1, A^{-}=\left(N_{c}^{2}-4\right) / N_{c}^{2}$ and

$$
\bar{P}_{g g}(z)=\frac{z}{(1-z)}+\frac{1-z}{z}+z(1-z)+\frac{b_{0}}{4 N_{c}} \delta(1-z), \quad \bar{P}_{g q}(z)=\frac{1+(1-z)^{2}}{z} .
$$

Our result does not agree with that of Ref. [9], Eqs. (109), (110) in an overall sign in front of the $T_{\Delta G, F}^{(f(d))}$ distribution (which may, however, be an artifact of a different sign convention for $\epsilon_{\mu \nu \alpha \beta}$ ), and two extra terms (up to the $\alpha_{s} N_{c} / \pi$ factor)

$$
T_{F}^{ \pm}(x, x) \pm \int_{x}^{1} \frac{d \xi}{\xi} \frac{1-z}{2}\left(T_{F}^{ \pm}(x, x-\xi)-\Delta T_{F}^{ \pm}(x, x-\xi)\right)
$$

which seem to have the same origin as the extra contributions that we also have for the flavor-nonsinglet distributions.

\section{Conclusions}

We have presented a complete reanalysis of the scale dependence of twist-three three-particle correlation functions that are relevant for calculations of single transverse spin asymmetries in the framework of collinear factorization. The calculation was done using the two-particle kernels for the renormalization of light-ray operators, which are available from Ref. [14]. Evolution equations can be obtained for arbitrary parton momentum fractions, for the flavor-(non)singlet distributions, with both positive and negative $C$-parity. Specializing to the case of zero gluon momentum we have compared our results with the recent calculation in Ref. [9]. There are two terms where we disagree, and their origin could be identified. Numerical studies of the evolution effects on realistic models of parton distributions can be found in Ref. [26].

\section{References}

1. M. Anselmino, A. Efremov and E. Leader, Phys. Rept. 261, 1 (1995) [Erratum-ibid. 281, 399 (1997)]. 
2. A. V. Efremov and O. V. Teryaev, Sov. J. Nucl. Phys. 36, 140 (1982).

3. A. V. Efremov and O. V. Teryaev, Phys. Lett. B 150, 383 (1985).

4. J. w. Qiu and G. Sterman, Phys. Rev. Lett. 67, 2264 (1991).

5. X. Ji, J. w. Qiu, W. Vogelsang and F. Yuan, Phys. Rev. Lett. 97, 082002 (2006).

6. X. Ji, J. w. Qiu, W. Vogelsang and F. Yuan, Phys. Rev. D 73, 094017 (2006).

7. X. Ji, J. w. Qiu, W. Vogelsang and F. Yuan, Phys. Lett. B 638, 178 (2006).

8. Y. Koike, W. Vogelsang and F. Yuan, Phys. Lett. B 659, 878 (2008).

9. Z. B. Kang and J. W. Qiu, Phys. Rev. D 79 (2009) 016003.

10. W. Vogelsang and F. Yuan, arXiv:0904.0410 [hep-ph].

11. A. P. Bukhvostov, E. A. Kuraev and L. N. Lipatov, JETP Lett. 37, 482 (1983); Sov. Phys. JETP 60, 22 (1984).

12. A. P. Bukhvostov, G. V. Frolov, L. N. Lipatov and E. A. Kuraev, Nucl. Phys. B 258 (1985) 601.

13. V. M. Braun, A. N. Manashov and J. Rohrwild, Nucl. Phys. B 807, 89 (2009).

14. V. M. Braun, A. N. Manashov and J. Rohrwild, arXiv:0908.1684 [hep-ph].

15. R. L. Jaffe, Nucl. Phys. B 229, 205 (1983).

16. I. I. Balitsky and V. M. Braun, Nucl. Phys. B 361, 93 (1991).

17. M. Diehl, Phys. Rept. 388, 41 (2003).

18. A. V. Belitsky and A. V. Radyushkin, Phys. Rept. 418, 1 (2005).

19. I. I. Balitsky and V. M. Braun, Nucl. Phys. B 311 (1989) 541.

20. D. Mueller, Phys. Lett. B 407, 314 (1997).

21. V. M. Braun, G. P. Korchemsky and A. N. Manashov, Phys. Lett. B 476, 455 (2000).

22. V. M. Braun, G. P. Korchemsky and A. N. Manashov, Nucl. Phys. B 603 (2001) 69.

23. V. M. Braun, A. N. Manashov and B. Pirnay, Phys. Rev. D 80, 114002 (2009).

24. Y. Koike and K. Tanaka, arXiv:0907.2797 [hep-ph].

25. A. Ali, V. M. Braun and G. Hiller, Phys. Lett. B 266, 117 (1991).

26. V. M. Braun, T. Lautenschlager, A. N. Manashov and B. Pirnay, Phys. Rev. D 83, 094023 (2011). 